\title{
Production and characterisation of mouse monoclonal antibodies reactive with the lipopolysaccharide core of Pseudomonas aeruginosa
}

\author{
J. W. NELSON, G. R. BARCLAY, L. R. MICKLEM* I. R. POXTON and J. R. W. GOVAN \\ Departments of Medical Microbiology and "Surgery, University of Edinburgh Medical School, Teviot Place, \\ Edinburgh EH8 9AG and +Regional Blood Transfusion Centre and Department of Transfusion Medicine, \\ Royal Infirmary, Edinburgh, Scotland
}

\begin{abstract}
Summary. Monoclonal antibodies (MAbs) to the core antigen region of lipopolysaccharide (LPS) of Pseudomonas aeruginosa were produced from mice immunised with whole cells of heat-killed rough mutants of Pseudomonas aeruginosa expressing partial or complete core LPS. MAbs were screened in an enzyme-linked immunosorbent assay (ELISA) against three different antigen cocktails : S-form LPS from three $P$. aeruginos $a$ strains, R-form LPS from six $P$. aeruginosa strains and, as a negative control, R-form LPS from Salmonella typhimurium and Escherichia coli. Selected MAbs were subsequently screened against a range of extracted LPS and whole cells from both reference strains and clinical isolates of $P$. aeruginosa. The antibodies were also screened in ELISA against whole-cell antigens from other Pseudomonas spp. as well as strains of Haemophilus influenzae, Neisseria subflava and Staphylococcus aureus. Five MAbs reacting with the core component of $P$. aeruginosa LPS were finally selected. Two of these, MAbs 360.7 and 304.1.4, were particularly reactive in immunoblots against unsubstituted core LPS, including that from O-antigenic serotypes of $P$. aeruginosa. The MAbs also reacted with some of the other Pseudomonas spp., but not with P. cepacia or Xanthomonas (Pseudomonas) maltophilia. Cross-reactivity with whole cells from other bacterial species was minimal or not observed. Reactivity of MAbs with some Staph. aureus strains was observed, and binding to the protein A component was implicated. The reactivity of the MAbs was investigated further by flow cytometry and immunogold electronmicroscopy. The suitability of the MAbs for an immunological assay for detection of $P$. aeruginosa in respiratory secretions from $\mathrm{CF}$ patients is discussed.
\end{abstract}

\section{Introduction}

Pseudomonas aeruginosa is an opportunist pathogen par excellence, responsible for a diverse range of infections in patients compromised by chemotherapy, injury, surgical procedures or underlying disease, and by the inherited disease cystic fibrosis (CF). Pulmonary colonisation of patients with CF by $P$. aeruginosa can be diagnosed by bacteriological culture and detection of pseudomonas-specific antibodies..$^{1-3}$ However, in the early stages of asymptomatic colonisation such techniques may be unhelpful.

There has been increasing interest in the development of immunoassays which can detect infective agents directly in clinical specimens and be adapted to the rapid screening of a large number of specimens. ${ }^{4-6}$ The development of an immunoassay based on a monoclonal antibody (MAb) for the early detection of $P$. aeruginosa antigens in saliva and sputum specimens from CF patients was considered a potentially useful diagnostic technique.

$P$. aeruginosa is serologically heterogeneous and contains at least 20 different heat-stable $\mathrm{O}$-antigens. ${ }^{?}$ The aim of the current study was to produce and characterise MAbs specific for $P$. aeruginosa and then assess their suitability for the detection of any of the distinct serotypes which may colonise individual $\mathrm{CF}$ patients. $P$. aeruginosa produces several intra- and extra-cellular antigens. Amongst these, lipopolysaccharide (LPS) is highly immunogenic and readily released from bacterial cells. ${ }^{89}$ Therefore, the common core region of LPS was considered to be an appropriate antigen for the production of anti- $P$. aeruginosa MAbs for use in an immunoassay to detect $P$. aeruginosa in clinical specimens from $C F$ patients.

\section{Materials and methods}

\section{Bacterial strains}

$P$. aeruginosa R-mutants defective in the production 
of LPS (PAC556, PAC557, PAC605, PAC608, PAC609 and PAC611) were obtained from Professor P. M. Meadow, University College, London. ${ }^{10}$ Standard serotype strains of $P$. aeruginosa were obtained from Dr R. J. Jones, University of Liverpool. The standard genetic strain $P$. aeruginosa PAO1 was also included in the study. Strains of $P$. acidovorans NCIB 9681, P. fluorescens NCIB 10525, P. mendocina NCIB 10541, P. pseudoalkaligenes NCIB 9946, P. putida NCIB 1007 , P. stutzeri NCIB 9040 and P. testosteroni NCIB 8893 were supplied by the National Collection of Industrial Bacteria. P. cepacia strains J1685, J1695, $\mathrm{J} 1705$, J1715, J1725, J1735, J1765 and J1775 were obtained from the Center for Communicable Disease Control, Atlanta, GA, USA. ${ }^{11}$ Bacteria denoted by the prefix JN were isolated from sputum of patients with CF and included strains of $P$. aeruginosa, $P$. cepacia, Xanthomonas (Pseudomonas) maltophilia, Haemophilus influenzae, $H$. parainfluenzae, Neisseria subflava, Moraxella (Branhamella) catarrhalis and Staphylococcus aureus. Enterobacterial strains used as controls included Ra mutants Escherichia coli R1 and Salmonella typhimurium 1542, and a strain of Proteus mirabilis.

\section{Immunisation procedure and production of MAbs}

$P$. aeruginosa was grown in nutrient broth containing yeast extract $0.5 \% \mathrm{w} / \mathrm{v}(\mathrm{NYB})$, washed twice with phosphate-buffered saline (PBS, $\mathrm{pH}$ 7.2) and resuspended to a density of $10^{8}$ cells $/ \mathrm{ml}$. Five female BALB/c mice were immunised by intravenous (i.v.) injection with $10^{7}$ heat-killed $\left(100^{\circ} \mathrm{C}\right.$ for $\left.5 \mathrm{~min}\right)$ cells. Each mouse received $0.1 \mathrm{ml}$ of cells from $P$. aeruginosa PAC608 (full core mutant) on days 1, 2, 3, 8, 9 and 10 followed by cells from $P$. aeruginosa PAC605 (partial core mutant) on days 22,23 and 24 . Blood was taken from the tail vein of each mouse on days 1 (preinoculation), 20,31 and 38, and the antibody response was measured by ELISA (see below). A mouse with serum showing a strong, cross-reactive antibody response to $P$. aeruginosa LPS antigens was selected for fusion. The mouse was given a booster injection of $0.1 \mathrm{ml}$ of heat-killed cells of PAC608 $\left(10^{7}\right.$ cells $)$, i.v., and was killed 3 days later. Mouse spleen cells were fused with cells of the NS-1 plasmacytoma cell line ${ }^{12}$ by the method of Oi and Herzenberg. ${ }^{13}$ Hybridomas producing anti- $P$. aeruginosa LPS antibodies were screened by ELISA (see below). Antibody-positive hybrids were cloned by limiting dilution.

\section{Preparation of LPS}

Extraction of LPS from bacterial strains expressing the S-form LPS phenotype was based on the aqueous phenol method of Westphal and Luderitz. ${ }^{14}$ The aqueous phenol, chloroform, petroleum ether method of Galanos et al. ${ }^{15}$ incorporating the diethyl ether precipitation of LPS described by Qureshi et al. ${ }^{16}$ (as described by Hancock and Poxton ${ }^{17}$ ), was used to prepare LPS from bacteria expressing the R-form LPS.

\section{Characterisation of MAbs}

Isotype determination. The class and subclass of MAbs in culture supernates were determined by a mouse MAb isotyping kit (RPN29: Amersham International plc).

Enzyme-linked immunosorbent assay (ELISA). An ELISA was used (a) to detect the antibody response of mice to vaccination; (b) to screen hybridomas for the production of anti- $P$. aeruginos $a$ antibody; and (c) to test the reactivity of MAbs against other bacterial strains. A primary ELISA screen consisting of three different LPS cocktails was used for an initial assessment of the reactivity of antibodies in sera from mouse-tail samples and in hybridoma supernates. Extracted LPS antigens were complexed with polymyxin as described by Scott and Barclay ${ }^{18}$ and antigen cocktails were formed by the addition of equimolar proportions of individual complexes. Cocktail-A consisted of O-antigenic S-form LPS from three serotype strains of $P$. aeruginosa $(\mathrm{O} 1, \mathrm{O} 2$ and $\mathrm{O} 11)$; cocktail-B consisted of core R-LPS from six LPS R-mutants (PAC556, PAC557, PAC605, PAC608, PAC609 and PAC611); cocktail-C (negative control) consisted of LPS from $S$. typhimurium 1542 and $E$. coli R1. Hybridomas producing a strong antibody response directed to one or both of the $P$. aeruginosa LPS cocktails were selected and the antibodies were subsequently tested in a secondary ELISA consisting of the individual LPS antigens contained in the LPS cocktails of the primary screen. Hybridomas producing antibodies of interest were further screened in ELISA against a comprehensive range of antigens which included additional LPS-polymyxin complexes, individual pseudomonas vaccine antigens PVA-1-16 which comprise the polyvalent pseudomonas extract vaccine (PEV) ${ }^{19}$ and whole-cell bacterial antigens. All antigens for ELISA studies were diluted in carbonatebicarbonate coating buffer ( $\mathrm{pH} \mathrm{9.6)}$ ) and added to the wells of polystyrene microplate 8-well strips (Nunc, 'polysorb') at $100 \mu \mathrm{l} /$ well. LPS-polymyxin complexes were diluted 1 in 50 in coating buffer and vaccine antigens were coated at $1 \mu \mathrm{g} / \mathrm{ml}$. Bacterial whole cells (grown overnight in NYB and washed twice with PBS) were resuspended to a density of $10^{7}$ cells $/ \mathrm{ml}$ in coating buffer and added to the microtitration plate wells, and the plates were centrifuged at $1400 \mathrm{~g}$ for $5 \mathrm{~min}$ to sediment bacteria to the wells. After washing with wash buffer (PBS containing Tween $200.05 \% \mathrm{v} / \mathrm{v}$ and sodium azide $0.02 \% \mathrm{w} / \mathrm{v}$ ), all plates were post-coated with post-coat buffer (PBS containing bovine serum albumin, BSA, $5 \% \mathrm{w} / \mathrm{v}$ ) at $100 \mu \mathrm{l} /$ well. After being washed four times with wash buffer, plates were stored at $-20^{\circ} \mathrm{C}$ until use.

Primary antibody (sera from mouse tail samples diluted 1 in 100 , or supernate from hybridomas diluted 1 in 5) was added $(100 \mu l)$ to each well. Antibody diluent was PBS with Tween $200.05 \% \mathrm{v} / \mathrm{v}$, BSA, 


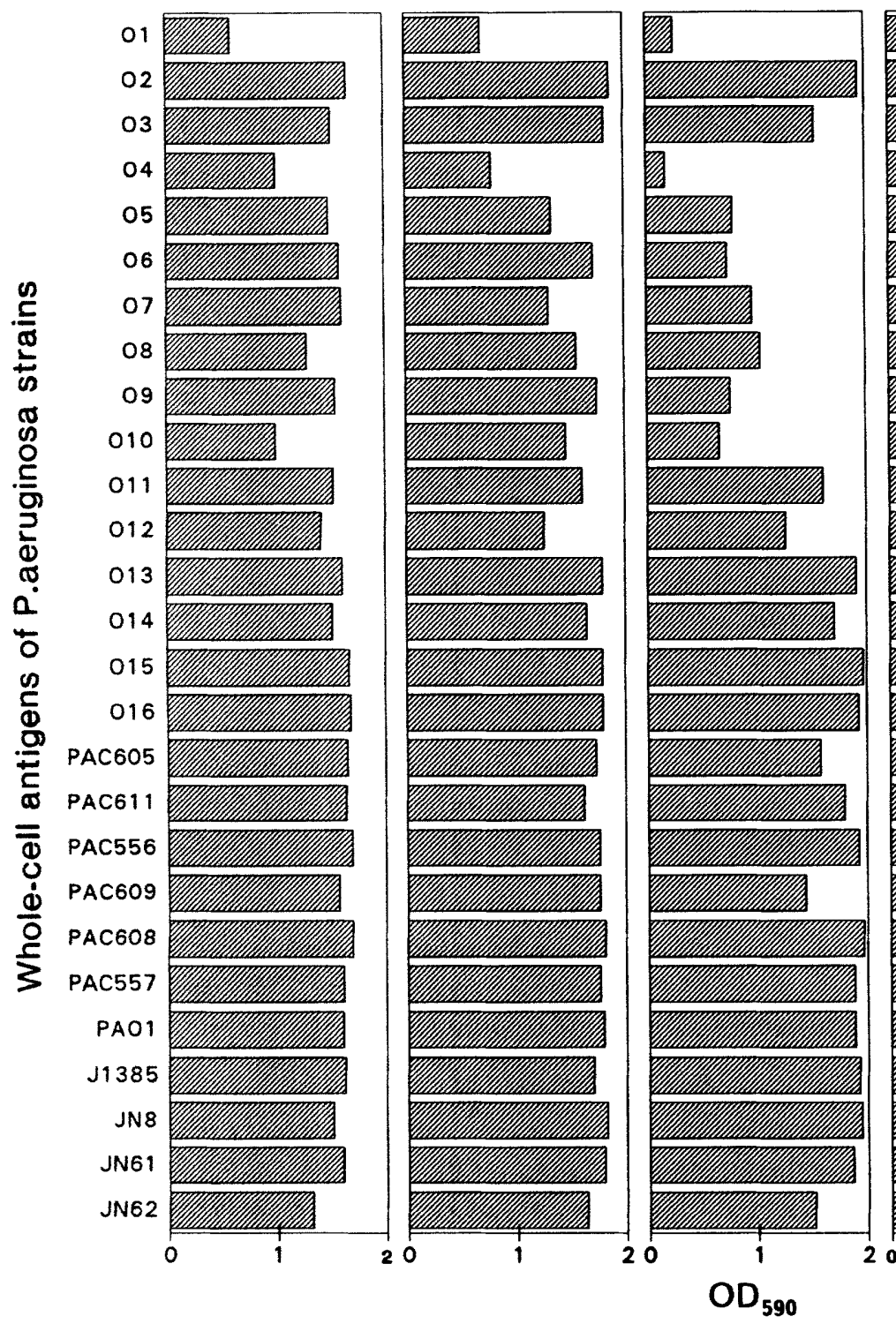

MAbs $\quad 304.1 .4$

61.3.2

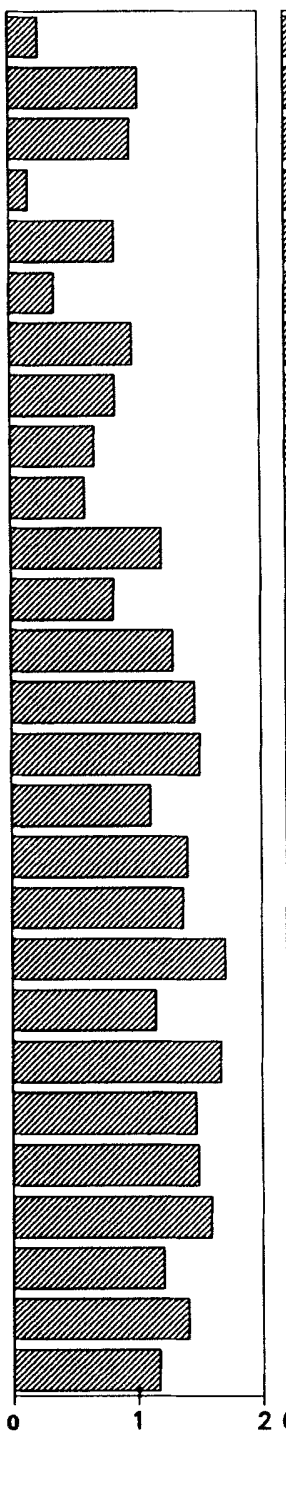

73.5

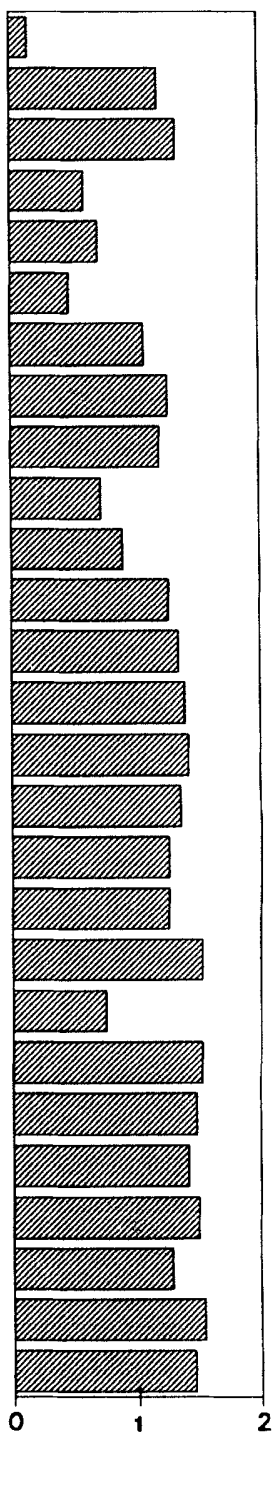

334.4

Fig. 1. Reactivity in ELISA of anti-LPS MAbs against $P$. aeruginosa whole cells of standard serotype strains O1-O16, R-LPS mutants, PAC605-PAC557, the standard genetic strain PA01 and clinical isolates from patients with CF, J1385-JN62.

$0.5 \% \mathrm{w} / \mathrm{v}$, polyethylene glycol $60004 \% \mathrm{w} / \mathrm{v}$ and sodium azide $0.02 \% \mathrm{w} / \mathrm{v}$. After incubation at $37^{\circ} \mathrm{C}$ for $90 \mathrm{~min}$, plates were washed four times with wash buffer. Urease-conjugated anti-mouse Ig (Sera Lab) was diluted 1 in 500 in diluent, and then $100 \mu \mathrm{l}$ was added to each well. The plates were incubated for a further $90 \mathrm{~min}$ at $37^{\circ} \mathrm{C}$. Thereafter they were washed and rinsed, and $100 \mu$ l of urease substrate (Sera Lab) was added to each well. Finally, the plates were incubated for $60 \mathrm{~min}$ at room temperature and the optical density (OD) of wells was read at $590 \mathrm{~nm}$ in a Titertek Multiscan. Results were expressed after subtraction of the corresponding OD of negative control wells (coated only with BSA) for each antibody sample.

Immunoblotting. LPS was prepared by the proteinase $\mathrm{K}$ method of Hitchcock and Brown ${ }^{20}$ and separated on polyacrylamide $14 \%$ gels with the buffer system of Laemmli ${ }^{21}$ but without sodium dodecyl sulphate in the stacking and separating gel buffers.
Separated antigens were transferred to nitrocellulose membranes (Schleicher and Schuell, pore size $0.2 \mu \mathrm{m}$ ) by the method of Towbin et $a l^{22}$ and processed by the method of Hancock and Poxton. ${ }^{17}$ Antigens were probed with hybridoma culture supernate diluted 1 in 5 for $3 \mathrm{~h}$ at room temperature and the immune complexes were detected with anti-mouse Ig horseradish peroxidase conjugate (ICN Biomedicals) and HRP colour reagent (BioRad).

Flow cytometry. The binding of MAbs (hybridoma supernates) to whole bacteria was analysed by the flow cytometry method described by Nelson et al. ${ }^{23}$

Immunogold electronmicroscopy. $P$. aeruginosa strains were grown overnight in NYB, and harvested by centrifugation at $10000 \mathrm{~g}$ for $10 \mathrm{~min}$. Cells for thin sections and the subsequent immunoassay were prepared as described by Hancock and Poxton. ${ }^{17}$ MAb supernates and the anti-mouse IgG immunogold conjugate (10 $\mathrm{nm}$ particle size, Sigma) were diluted 1 in 10 in antibody diluent which was the same as for the 
Table I. Cross-reactivity in ELISA of anti-LPS MAbs against bacterial whole cells

\begin{tabular}{|c|c|c|c|c|c|c|}
\hline \multirow{2}{*}{$\begin{array}{c}\text { Bacterial species } \\
\text { tested }\end{array}$} & \multirow{2}{*}{$\begin{array}{c}\text { Number } \\
\text { of strains } \\
\text { tested }\end{array}$} & \multicolumn{5}{|c|}{ Percentage antibody binding relative to $P$. aeruginosa* } \\
\hline & & 304.14 & 360.7 & 61.32 & 73.5 & 334.4 \\
\hline P. aeruginosa & 21 & 100 & 100 & 100 & 100 & 100 \\
\hline$P$. mendocina & 2 & 56 & 89 & 48 & 100 & 44 \\
\hline P. fluorescens & 3 & 78 & 84 & 47 & 69 & 65 \\
\hline P. pseudoalkaligenes & 1 & 56 & 66 & 13 & 95 & 87 \\
\hline P. acidovorans & 1 & 83 & 85 & 0 & 35 & 85 \\
\hline P. putida & 4 & 86 & 73 & 64 & 100 & 100 \\
\hline P. stutzeri & 1 & 77 & 87 & 30 & 86 & 78 \\
\hline P. testosteroni & 1 & 0 & $\mathbf{0}$ & 0 & 93 & 0 \\
\hline P. cepacia & 10 & 0 & 0 & 0 & 0 & 0 \\
\hline X. maltophilia & 7 & 0 & 7 & 12 & 0 & 0 \\
\hline H. influenzae & 5 & 0 & 0 & 0 & 0 & 0 \\
\hline H. parainfluenzae & 2 & 0 & 6 & 5 & 0 & 0 \\
\hline N. subflava & 2 & 0 & 0 & 0 & 0 & 0 \\
\hline M.(B.) catarrhalis & 2 & 0 & 0 & 0 & 0 & 0 \\
\hline Staph. aureus & 4 & 32 & 29 & 33 & 50 & 38 \\
\hline E. coli $\mathrm{R} 1$ & 1 & 0 & 0 & 0 & 0 & 0 \\
\hline S. typhimurium 1542 & 1 & 0 & 0 & 0 & 0 & 0 \\
\hline Pr. mirabilis & 1 & 0 & 0 & 0 & 0 & 0 \\
\hline
\end{tabular}

* Percentage antibody binding values represent the mean $\mathrm{OD}_{590}$ relative to the mean $\mathrm{OD}_{590}$ calculated for $P$. aeruginosa.

ELISA studies. Sections were examined before and after staining with aqueous uranyl acetate, in a Hitachi $12 \mathrm{~A}$ electronmicroscope at $75 \mathrm{kV}$.

Protein $A$ assay. The ELISA for detection of protein A was performed as described by Warnes et al. ${ }^{24}$

\section{Results}

A total of 530 hybridoma supernates were screened in ELISA; 42 hybridomas produced an ELISA OD of $>0.5$ in the initial screening against the $P$. aeruginosa R- and S-LPS cocktails. Antibody-positive hybrids were cloned and five hybridomas that produced specific antibody were characterised further.

\section{Isotype determination}

One MAb (73.5) was classified as $\mathrm{IgG}_{1}$, two MAbs (304.1.4 and 360.7) as $\operatorname{IgG}_{2 b}$ and two $\mathrm{MAbs}$ as $\mathrm{IgG}_{3}$ (61.32 and 334.4).

\section{Reaction in ELISA}

The five MAbs selected for study produced a positive response against all the $P$. aeruginosa wholecell antigens used in the screening (fig. 1). A strong reaction (ELISA OD $>1.0$ ) against most of the $P$. aeruginosa whole-cell antigens was particularly marked for MAbs 304.1.4 and 360.7. The MAbs also showed reactions with whole cells from other Pseudomonas spp. (table I). All five MAbs reacted with the whole cells of $P$. fluorescens, $P$. mendocina, $P$. pseudoalkaligenes, $P$. putida and $P$. stutzeri. All the MAbs except 61.3.2 produced a positive reaction with $P$. acidovorans, but only MAb 73.5 produced a detectable reaction with $P$. testosteroni. MAb 304.1.4 failed to show a detectable reaction with any of the $P$. cepacia or X. maltophilia strains, whereas MAbs 73.5 and 334.4 produced weak reactions with individual $X$. maltophilia strains. MAbs 360.7 and 61.3.2 also failed to produce a positive reaction with any of the $P$. cepacia strains, although weak reactions were obtained with some of the $X$. maltophilia strains. None of the MAbs reacted with whole cells of $H$. influenzae, $H$. parainfluenzae, E. coli R1, S. typhimurium 1542, Pr. mirabilis, N. subflava and M.(B) catarrhalis. Positive reactions were observed with Staph. aureus strains JN91 and JN92 which were subsequently identified as strong producers $(>25 \mathrm{ng} / \mathrm{ml})$ of protein A.

Confirmation of the cross-reactive nature of the five MAbs with all serotypes of $P$. aeruginosa were also provided by the positive response of each $\mathrm{MAb}$ to the cell-wall vaccine antigens (PVA), serotypes O1-O16 (fig. 2). The strongest reactions were again observed with MAbs 304.1.4 and 360.7. The MAbs also reacted with each of the extracted $P$. aeruginosa LPS antigens. In ELISA, the response of the five MAbs to core RLPS from the PAC mutants tended to be lower towards the shortest core structure PAC605 and the complete core structures PAC557 and PAC608. The antibodies did not react with the enterobacterial R-LPS antigens from E. coli R1 and S. typhimurium 1542.

\section{Immunoblotting}

The immunoblot of MAb 360.7 is shown in fig. 3. A positive reactive band corresponding to the low- $\mathrm{M}_{\mathrm{r}}$ core glycolipid of the $P$. aeruginosa LPS preparations was observed. The apparent absence of bands corresponding to the high- $M_{r}$ LPS of serotype strains indicated that the MAbs were unable to recognise core LPS substituted with O-antigenic side chains. However, a very faint ladder pattern corresponding to the high-M $M_{r}$ LPS of serotypes O4 and O6 (tracks 4 and 6 


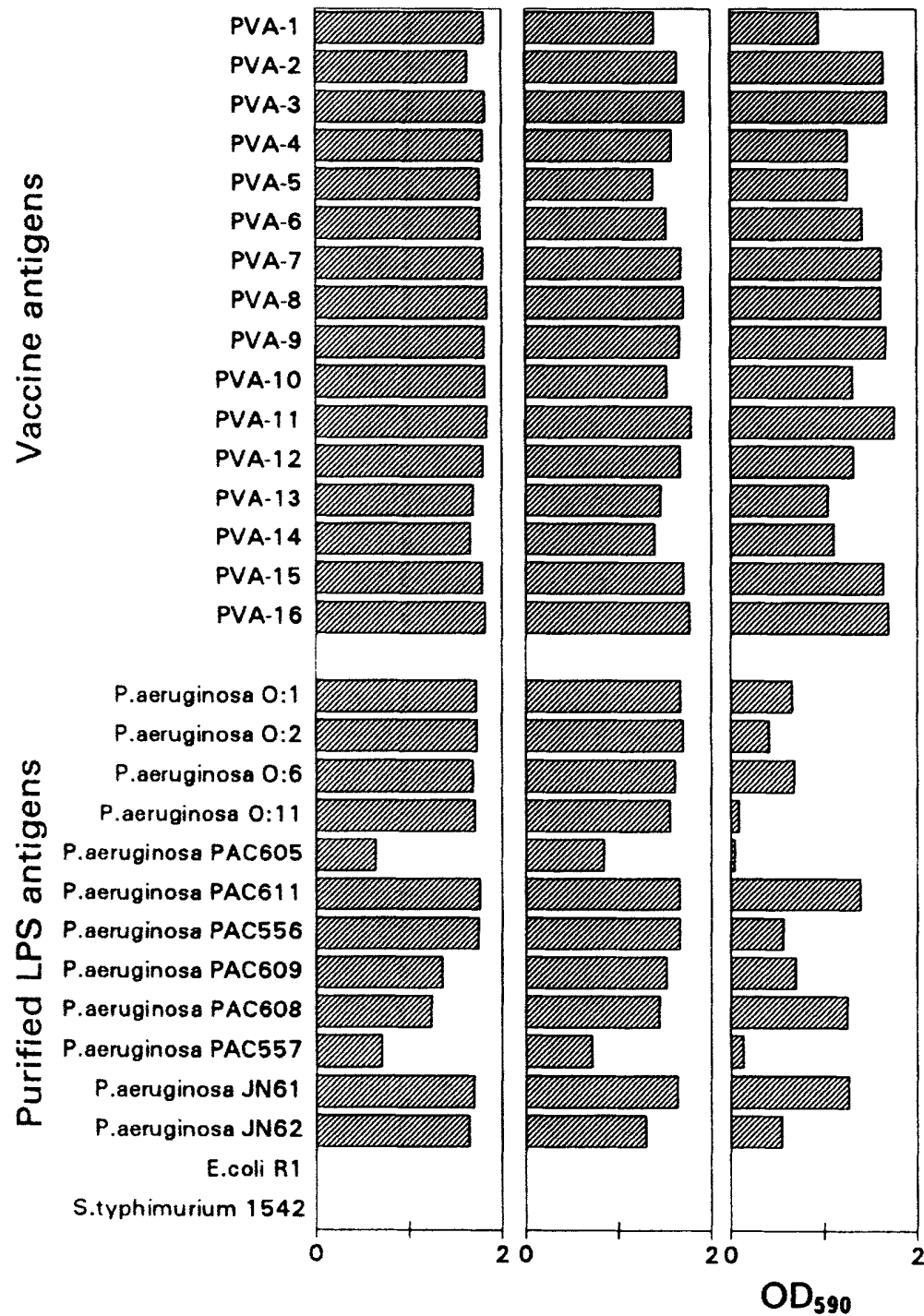

MAbs 304.1.4
360.7
61.3.2

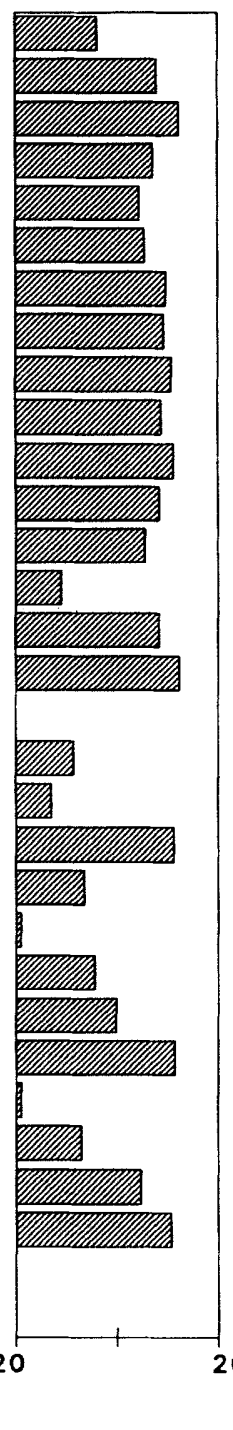

73.5

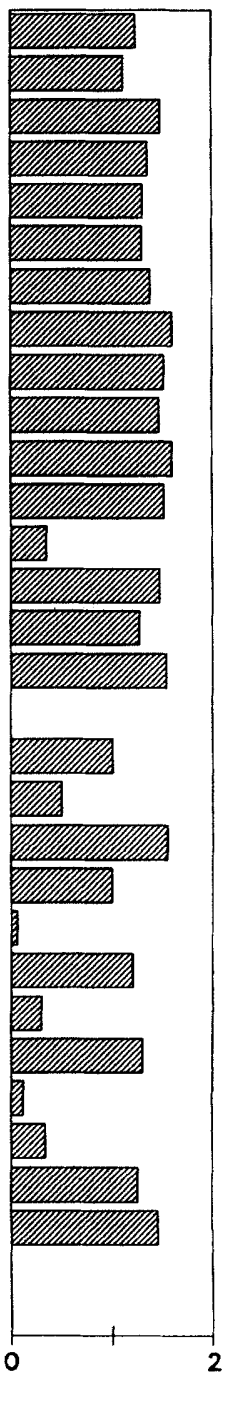

334.4

Fig. 2. Reactivity in ELISA of anti-LPS MAbs against $P$. aeruginosa S-LPS O-serotype vaccine components (PVA-1-16), extracted LPS antigens (LPS complexed with polymyxin) including S-LPS (O1-O11) and R-LPS (PAC605-PAC557), LPS from two P. aeruginosa strains (JN61 and JN62) from patients with CF, and LPS from two Enterobacteriaceae strains (negative controls).

respectively) was observed with MAb 360.7. The ladder patterns are barely visible on the photograph shown. The immunoblots obtained with MAbs 61.3.2, 73.5 and 334.4 were all weaker than the immunoblots obtained with MAbs 304.1.4 and 360.7. However, a positive reaction with the core components of most of the different LPS preparations was observed (data not shown)

\section{Flow cytometry on whole bacteria}

The binding of MAbs 304.1.4, 360.7 and 334.4 to whole $P$. aeruginosa cells was analysed by flow cytometry. The highest percentage values of bacteria exhibiting positive fluorescence were observed for the LPS R-mutants that expressed core or partial core structures. The MAbs also reacted with $P$. aeruginosa whole cells expressing O-antigen LPS. No reactivity was observed with whole cells of $P$. cepacia or the enterobacterial R-mutants $E$. coli $\mathrm{R} 1$ and $S$. typhimurium 1542 (table II).

\section{Immunogold electronmicroscopy}

Immunogold electronmicroscopy was also used to assess the binding of MAbs to $P$. aeruginosa cells processed for thin-sections. Fig. $4 \mathrm{a}$ and $\mathrm{b}$ shows the binding of MAb 360.7 to $P$. aeruginosa PAC611, which expresses only a partial core LPS, and to $P$. aeruginosa serotype O1. There was negligible binding of the MAbs to cells of $P$. cepacia (data not shown).

\section{Discussion}

The aim of this investigation was to prepare MAbs suitable for an immunoassay for the detection of nonmucoid strains of $P$. aeruginosa. MAbs recognising $P$. aeruginosa core LPS unsubstituted by O-serotype antigens were isolated and characterised. The MAbs were shown to be cross-reactive with core LPS antigens belonging to a variety of reference and clinical $P$. aeruginosa strains. The pan-reactive core-specific response of the MAbs, particularly MAbs 304.1.4 and 


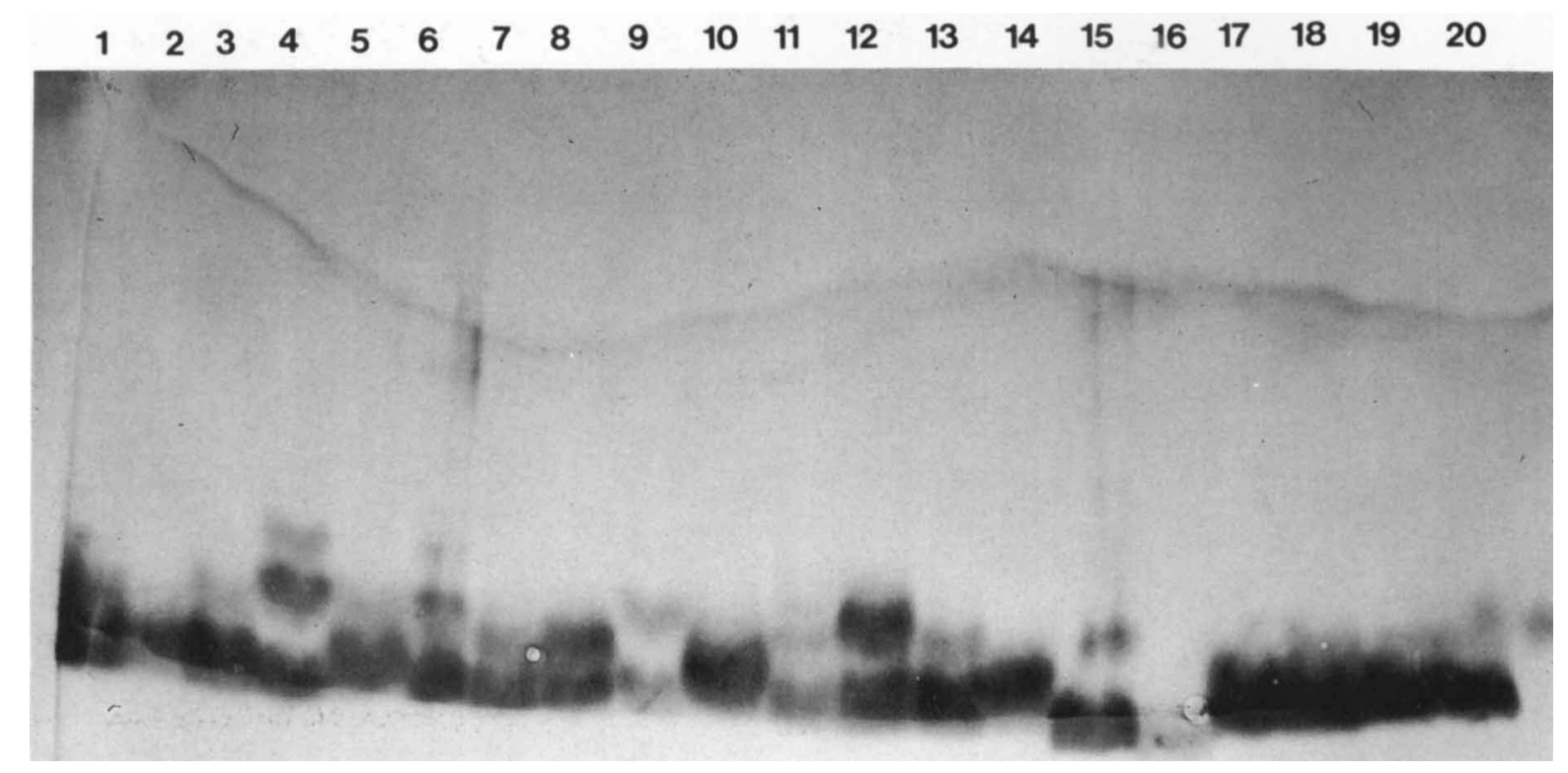

Fig. 3. Immunoblot of proteinase $\mathrm{K}$ whole-cell digests of $20 \mathrm{P}$. aeruginos $a$ strains separated by SDS-PAGE followed by transfer to nitrocellulose paper and probed with MAb 360.7 (supernate diluted 1 in 5). The immunoblot profiles against $P$. aeruginosa serotype strains O1, O2, O3, O4, O5, O6, O7, O8, O9, O10, O11, O12, O13, O15, and P. aeruginosa LPS R-mutants PAC611, PAC605, PAC609, PAC557 and PAC608 are shown in tracks $1-20$ respectively.

Table II. Reactivity in flow cytometry of anti-LPS MAbs against whole bacterial cells.

\begin{tabular}{llll}
\hline & \multicolumn{3}{c}{ Percentage reactivity* of MAb } \\
\cline { 2 - 4 } Strain & 304.1 .4 & 360.7 & 334.4 \\
\hline tested & & & \\
LPS-defective mutants & 71 & 80 & 75 \\
P. aeruginosa PAC605 & 20 & 50 & 18 \\
P. aeruginosa PAC611 & 40 & 73 & 40 \\
P. aeruginosa PAC556 & 13 & 64 & 52 \\
P. aeruginosa PAC609 & 24 & 87 & 60 \\
P. aeruginosa PAC608 & 21 & 39 & 40 \\
P. aeruginosa PAC557 & & & \\
Serotype strains & 28 & 31 & 27 \\
P. aeruginosa 01 & 30 & 45 & 31 \\
P. aeruginosa 02 & 30 & 52 & 28 \\
P. aeruginosa 06 & 37 & 51 & 41 \\
P. aeruginosa 09 & 32 & 31 & 29 \\
P. aeruginosa 011 & & & \\
Negative controls & 0 & 0 & 0 \\
P. cepacia JN93 & 0 & 0 & 0 \\
E. coli R1 & 0 & 0 & 0 \\
S. typhimurium 1542 & & & \\
\hline
\end{tabular}

*Values represent percentage of bacteria exhibiting positive fluorescence above background levels.

360.7 , supports the idea of the presence of a common antigenic structure in the core region of $P$. aeruginosa LPS. The inner core region of $P$. aeruginosa LPS is believed to be homogeneous and also common to most gram-negative bacteria, whereas the outer core region is considered to be heterogeneous ${ }^{25,26}$ and different from that of other gram-negative genera. ${ }^{27}$ Failure of the MAbs to react with LPS from $E$. coli R1 or $S$. typhimurium 1542, both of which express an Ra core LPS chemotype (complete core), or with whole cells of enterobacteria demonstrate that the MAbs generated recognise core epitopes in $P$. aeruginosa LPS which are not present in these selected strains of Enterobacteriaceae.

Failure of the MAbs to react with the ladder pattern of core LPS substituted with O-antigen, as shown by the immunoblot analysis, suggested that the epitope recognised was present at the distal end of the unsubstituted core oligosaccharide which became unavailable when $\mathrm{O}$-polysaccharide was linked. The fact that the MAbs showed strong reactions in ELISA and flow cytometry with $P$. aeruginosa whole cells expressing S-LPS antigen indicated that there was sufficient unsubstituted core glycolipid to allow binding by the MAbs. This may be a reflection of the low capping frequency of $P$. aeruginosa LPS (the percentage of core LPS constituents covered with Oantigen) leaving a large percentage of core determinants exposed at the cell surface. ${ }^{25,27,28}$ In contrast, however, this low level of covering with O-polymer 

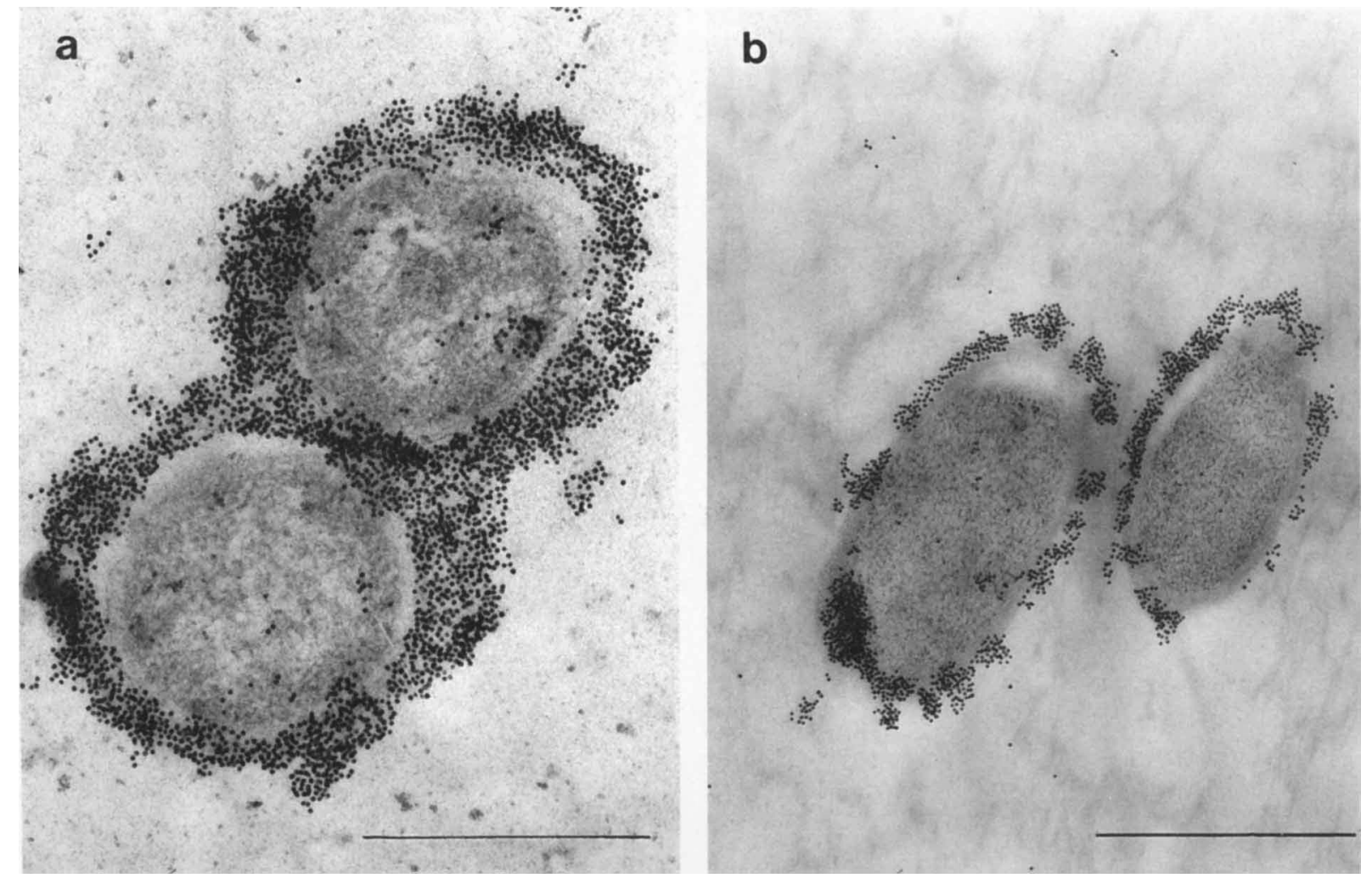

Fig. 4. Electronmicrographs of thin sections from cells of $P$. aeruginosa serotype $\mathrm{O} 1$ (a) and $P$. aeruginosa PAC61l (b) prepared by reaction with $\mathrm{MAb} 360.7$, followed by anti-mouse IgG-gold conjugate. Bars, $1 \mu \mathrm{m}$.

was able to inhibit the binding of the rough-core specific MAbs characterised by Sadoff et al..$^{29}$ MAbs $304.1 .4,360.7$ and 334.4 produced a reaction in ELISA, immunoblotting and flow cytometry with all the core mutants which indicated binding to a common epitope.

The MAbs investigated in this study were shown to react with various forms of $P$. aeruginosa LPS, including extracted LPS preparations, cell-wall extracts and whole bacterial cells, including those immobilised on microtitration-plate wells and those prepared for flow cytometry.

Cross-reactivity of the MAbs with whole cells representing a number of other Pseudomonas spp. indicates that these species contain core LPS similar in composition and structure to $P$. aeruginosa. Alternatively, their LPS may be structurally dissimilar, but serologically cross-reactive with that of $P$. aeruginosa. The absence of a significant response to (formerly Pseudomonas) $X$. maltophilia confirms the observations of Neal and Wilkinson ${ }^{30}$ who reported similarities between the LPS of $P$. maltophilia and that of some Xanthomonas spp. The lack of a significant response to $P$. cepacia may also reflect some of the reported differences in the structure of $P$. cepacia LPS. ${ }^{31}$

MAbs against $P$. aeruginosa core LPS and reactive with some ${ }^{26}$ or all serotypes have been reported previously. ${ }^{32}$ The MAbs described in this study are LPS-specific, pan-reactive with all $P$. aeruginosa regardless of serotype and, therefore, potentially useful for the detection of any of the serotype strains which may colonise individual $\mathrm{CF}$ patients. Recent studies have focused on the incorporation of the MAbs into a rapid immunological assay for the early diagnosis of asymptomatic colonisation of the $\mathrm{CF}$ respiratory tract. False positive reaction caused by the detection of $H$. influenzae, $X$. maltophilia, P. cepacia, Neisseria spp. and $M .(B$.$) catarrhalis, any of which may be$ found in CF respiratory secretions, are unlikely, given the fact that the MAbs failed to react significantly with whole cells of these organisms. However, the MAbs demonstrated reactivity with strong protein A-producing Staph. aureus cells which was probably caused by the interaction of protein A with the Fc portion of antibodies. Since Staph. aureus is commonly found in respiratory secretions of patients with $\mathrm{CF}$, any false positive reactions may have to be eliminated from an immunoassay for the detection of $P$. aeruginosa by pre-incubation with an Ig of a different non-crossreactive species.

This work (Project 3.3) was kindly supported by the Cystic Fibrosis Research Trust. We thank W. Neill for his expertise with the EPICS flow cytometer, and D. Notman, Oonagh Gray and Fiona Denison for assistance with the immunogold electronmicroscopy

\section{References}

1. Brett MM, Ghoneim ATM, Littlewood JM, Losowsky MS Development of enzyme linked immunosorbent assay (ELISA) to detect antibodies to Pseudomonas aeruginosa cell surface antigens in sera of patients with cystic fibrosis. $J$ Clin Pathol 1986; 39 : 1124-1129. 
2. Pedersen SS, Espersen F, Høiby NN. Diagnosis of chronic Pseudomonas aeruginosa infection in cystic fibrosis by enzyme-linked immunosorbent assay. J Clin Microbiol 1987; 25 : 1830-1836.

3. Nelson JW, Barclay GR, Govan JRW. Diagnosis of chronic Pseudomonas aeruginosa infection in cystic fibrosis by ELISA for anti-pseudomonas LPS IgG antibodies. Serodiag Immunother Infect Dis 1990; 4: 9-16.

4. Yolken RH. Enzyme immunoassays for the detection of infectious antigens in body fluids: current limitations and future prospects. Rev Infect Dis 1982: 4: 35-68.

5. Sugasawara RJ, Prato CM, Sippel JE. Enzyme-linked immunosorbent assay with a monoclonal antibody for detecting group A meningococcal antigens in cerebrospinal fluid. $J$ Clin Microbiol 1984; 19 : 230-234.

6. Gustafsson B, Askelöf P. Monoclonal antibody-based sandwich enzyme-linked immunosorbent assay for detection of Bordetella pertussis filamentous haemagglutinin. $J$ Clin Microbiol 1988; 26: 2077-2082.

7. Liu PV, Wang S. Three new major somatic antigens of Pseudomonas aeruginosa. J Clin Microbiol 1990; 28: 922-925.

8. Cadieux JE, Kuzio J, Milazzo FH, Kropinski AM. Spontaneous release of lipopolysaccharide by Pseudomonas aeruginosa. $J$ Bacteriol 1983: 155: 817-825

9. Kusama H. Enzyme-linked immunosorbent assay for detection of Pseudomonas aeruginosa lipopolysaccharides. J Clin Microbiol 1983: 17: 317-322.

10. Rowe PSN, Meadow PM. Structure of the core oligosaccharide from the lipopolysaccharide of Pseudomonas aeruginosa PAC1R and its defective mutants. Eur $J$ Biochem 1983; 132: $326-337$.

11. Rabkin CS, Jarvis WR, Anderson RL et al. Pseudomonas cepacia typing systems: collaborative study to assess their potential in epidemiologic investigations. Rev Infect Dis $1989 ; 11: 600-607$.

12. Kohler G, Howe SC, Milstein C. Fusion between immunoglobulin-secreting and nonsecreting myeloma cell lines. Eur J Immunol 1976; 6: 292-295.

13. Oi VT, Herzenberg LA. Immunoglobulin-producing hybrid cell-lines. In : Mishell BB, Shiigi SM (eds) Selected methods in cellular immunology. San Francisco, WH Freeman and Co. 1980: 351-372.

14. Westphal O, Luderitz O. Chemische erforschung von lipopolysdacchariden gramnegativer bacterien. Angew Chem $1954 ; 66: 407-417$.

15. Galanos C, Lüderitz O, Westphal O. A new method for the extraction of R lipopolysaccharides. Eur J Biochem 1969; 9: 245-249.

16. Qureshi N, Takayama K, Ribi E. Purification and structural determination of nontoxic lipid A obtained from the lipopolysaccharide of Salmonella typhimurium. J Biol Chem 1982; 257: 11808-11815.

17. Hancock IC, Poxton IR. Bacterial cell surface techniques. Chichester, John Wiley and Sons. 1988.
18. Scott BB, Barclay GR. Endotoxin-polymyxin complexes in an improved enzyme-linked immunosorbent assay for IgG antibodies in blood donor sera to gram-negative endotoxin core glycolipids. Vox Sang 1987; 52: 272-280.

19. Miler JM, Spilsbury JF, Jones RJ, Roe EA, Lowbury EJL. A new polyvalent Pseudomonas vaccine. $J$ Med Microbiol $1977 ; 10: 19-27$.

20. Hitchcock PJ, Brown TM. Morphological heterogeneity among Salmonella lipopolysaccharide chemotypes in silver-stained polyacrylamide gels. $J$ Bacteriol 1983 ; 154 : 269-277.

21. Laemmli UK. Cleavage of structural proteins during the assembly of the head of bacteriophage T4. Nature 1970; 227: 680-685.

22. Towbin H, Staehelin T, Gordon J. Electrophoretic transfer of proteins from polyacrylamide gels to nitrocellulose sheets: procedure and some applications. Proc Natl Acad Sci USA $1979 ; 76: 4350-4354$.

23. Nelson D, Neill W, Poxton IR. A comparison of immunoblotting, flow cytometry and ELISA to monitor the binding of anti-lipopolysaccharide monoclonal antibodies. $J$ Immunol Methods 1990; 133: 227-233.

24. Warnes A, Walkland A, Stephenson JR. Development of an enzyme immunosorbent assay for staphylococcal protein $A$ produced in Escherichia coli by pUC8 based plasmids containing the Staphylococcus aureus Cowan I protein A gene. J Immunol Methods 1986; 93: 63-70.

25. Hancock REW, Mutharia LM, Chan L, Darveau RP, Speert DP, Pier GB. Pseudomonas aeruginosa isolates from patients with cystic fibrosis: a class of serum-sensitive, nontypable strains deficient in lipopolysaccharide $\mathrm{O}$ side chains. Infect Immun 1983; 42: 170-177.

26. Yokota SI, Ochi $\mathrm{H}$, Ohtsuka $\mathrm{H}$, Kato $\mathbf{M}$, Noguchi $\mathrm{H}$. Heterogeneity of the L-rhamnose residue in the outer core of Pseudomonas aeruginosa lipopolysaccharide, characterized by using human monoclonal antibodies. Infect Immun 1989; 57: 1691-1696.

27. Wilkinson SG. Composition and structure of lipopolysaccharides from Pseudomonas aeruginosa. Rev Infect Dis 1983; 5 Suppl 5: S941-S949.

28. Rivera M, Bryan LE, Hancock REW, McGroarty EJ. Heterogeneity of lipopolysaccharides from Pseudomonas aeruginosa: analysis of lipopolysaccharide chain length. $J$ Bacteriol 1988; 170: 512-521.

29. Sadoff JC, Wright DC, Futrovsky S, Sidberry $\mathbf{H}$, Collins $H$, Kaufmann B. Characterization of mouse monoclonal antibodies directed against Pseudomonas aeruginosa lipopolysaccharides. Antibiot Chemother 1985; 36: 134-146.

30. Neal DJ, Wilkinson SG. Lipopolysaccharides from Pseudomonas maltophilia. Structural studies of the side-chain, core, and lipid-A regions of the lipopolysaccharide from strain NCTC 10257. Eur J Biochem 1982; 128: 143-149.

31. Cox AD, Wilkinson SG. Ionizing groups in lipopolysaccharides of Pseudomonas cepacia in relation to antibiotic resistance. Mol Microbiol 1991; 5: 641-646.

32. Gaston MA, Vale TA, Wright B, Cox P, Pitt TL. Monoclonal antibodies to the surface antigens of Pseudomonas aeruginosa. FEMS Microbiol Lett 1986; 37: 357--361. 